\title{
A UK general practice population cohort study investigating the association between lipid lowering drugs and 30-day mortality following medically attended acute respiratory illness
}

Roshni Joshi, Sudhir Venkatesan, Puja R Myles

Background. Cholesterol lowering drugs HMG-CoA reductase inhibitors (statins) and PPAR $\alpha$ activators (fibrates) have been shown to reduce host inflammation via non-disease specific immunomodulatory mechanisms. Recent studies suggest that commonly prescribed drugs in general practice, statins and fibrates, may be beneficial in influenzalike illness related mortality. This retrospective cohort study examines the association between two lipid lowering drugs, statins and fibrates, and all-cause 30-day mortality following a medically attended acute respiratory illness (MAARI).

Methods. Primary care patient data were retrospectively extracted from the UK Clinical Practice Research Datalink (CPRD) database. The sample comprised 201,179 adults aged 30 years or older experiencing a MAARI episode. Patient exposure to statins or fibrates was coded as separate dichotomous variables and deemed current if the most recent GP prescription was issued in the 30 days prior to MAARI diagnosis. Multivariable logistic regression and Cox regression was used for analyses. Adjustment was carried out for chronic lung disease, heart failure, metformin and glitazones, comorbidity burden, sociodemographic and lifestyle variables such as smoking status and body mass index (BMI). Statistical interaction tests were carried out to check for effect modification by gender, body mass index, smoking status and comorbidity.

Results. A total of 1,096 (5\%) patients died within the 30 -day follow up period. Of this group, $213(19.4 \%)$ were statin users and $4(0.4 \%)$ were fibrate users. After adjustment, a significant 35\% reduction in odds [adj OR; $0.65(95 \% \mathrm{Cl} 0.52-0.80)$ ] and a $33 \%$ reduction in the hazard [adj HR: $0.67(95 \% \mathrm{Cl} 0.55-0.83)$ ] of all-cause 30-day mortality following MAARI was observed in statin users. A significant effect modification by comorbidity burden was observed for the association between statin use and MAARI-related mortality. Fibrate use was associated with a non-significant reduction in 30-day MAARI-related mortality. 
Conclusion. This study suggests that statin use may be associated with a reduction in 30day mortality following acute respiratory illness that is severe enough to merit medical consultation. Findings from this study support and strengthen similar observational research while providing a strong rationale for a randomised controlled trial investigating the potential role of statins in acute respiratory infections. 
1 A UK general practice population cohort study investigating

2 the association between lipid lowering drugs and 30-day

3 mortality following medically attended acute respiratory

4 illness

5

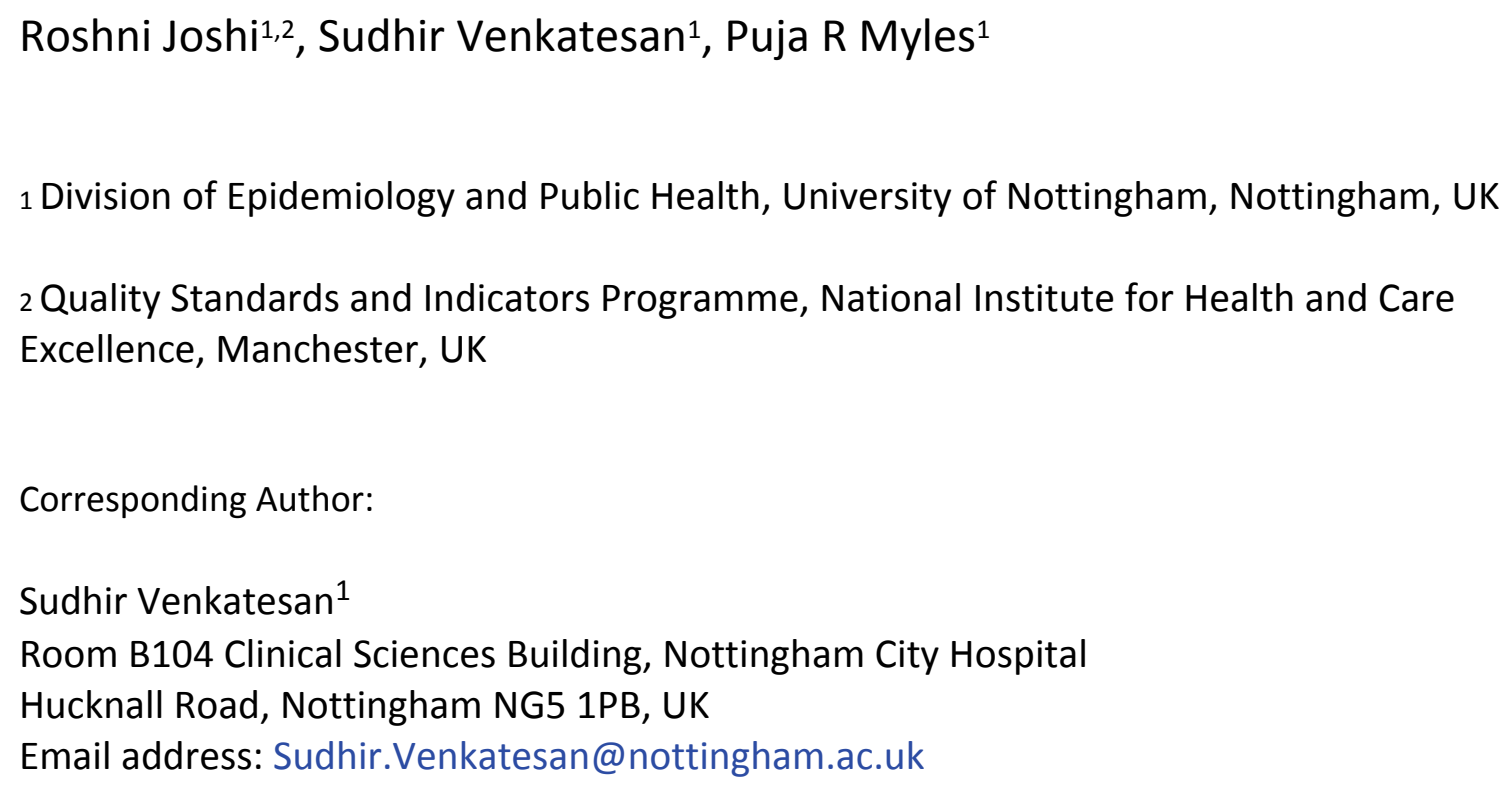

Running title: Lipid lowering drugs and ARI related death 
46 Abstract

47 Background

48 Cholesterol lowering drugs HMG-CoA reductase inhibitors (statins) and PPAR $\alpha$ activators 49 (fibrates) have been shown to reduce host inflammation via non-disease specific 50 immunomodulatory mechanisms. Recent studies suggest that commonly prescribed drugs in 51 general practice, statins and fibrates, may be beneficial in influenza-like illness related mortality. 52 This retrospective cohort study examines the association between two lipid lowering drugs, 53 statins and fibrates, and all-cause 30-day mortality following a medically attended acute 54 respiratory illness (MAARI).

\section{Methods}

56 Primary care patient data were retrospectively extracted from the UK Clinical Practice Research 57 Datalink (CPRD) database. The sample comprised 201,179 adults aged 30 years or older 58 experiencing a MAARI episode. Patient exposure to statins or fibrates was coded as separate 59 dichotomous variables and deemed current if the most recent GP prescription was issued in the 6030 days prior to MAARI diagnosis. Multivariable logistic regression and Cox regression were 61 used for analysis. Adjustment was carried out for chronic lung disease, heart failure, metformin 62 and glitazones, comorbidity burden, socio-demographic and lifestyle variables such as smoking 63 status and body mass index (BMI). Statistical interaction tests were carried out to check for 64 effect modification by gender, body mass index, smoking status and comorbidity.

65 Results

66 A total of 1,096 (5\%) patients died within the 30-day follow up period. Of this group, 213 $67(19.4 \%)$ were statin users and $4(0.4 \%)$ were fibrate users. After adjustment, a significant 35\% 
68 reduction in odds [adj OR; 0.65 (95\% CI 0.52-0.80)] and a 33\% reduction in the hazard [adj HR:

$690.67(95 \%$ CI $0.55-0.83)]$ of all-cause 30-day mortality following MAARI was observed in statin

70 users. A significant effect modification by comorbidity burden was observed for the association

71 between statin use and MAARI-related mortality. Fibrate use was associated with a non-

72 significant reduction in 30-day MAARI-related mortality.

\section{Conclusions}

74 This study suggests that statin use may be associated with a reduction in 30-day mortality

75 following acute respiratory illness that is severe enough to merit medical consultation. Findings

76 from this study support and strengthen similar observational research while providing a strong

77 rationale for a randomised controlled trial investigating the potential role of statins in acute

78 respiratory infections.

79 (Maximum word count: 350 words; Current word count: 346 words)

80 Keywords: lipid lowering drugs, statins, fibrates, acute respiratory illness, MAARI, mortality,

81 cohort study, CPRD

82

83

84

85

86

87

88

89

90

91

92

93

94

95

96

97

98

99 
100

101

102

103

104

105

106

107

108

109

110

111

112

113

114 115 been reported following their administration in hypercholesterolaemic and

\section{Introduction}

Globally, an estimated 3.9 million people are killed annually due to acute respiratory infections (ARI). Moreover, specific respiratory infections such as influenza and respiratory syncytial virus (RSV) are major contributors to the mortality and burden from ARI[1]. Statins are competitive inhibitors of 3-hydroxy-3-methylglutaryl coenzyme A (HMG-CoA) reductase and are used as lipid lowering drugs to reduce plasma cholesterol[2] and a reduction in chronic inflammation has normocholesterolaemic individuals[3]. In vivo studies demonstrating the ability of statins to

117 repress MHC-II expression, inhibit $\mathrm{T}$ cell activation, and limit the release of pro-inflammatory 118 cytokines further suggest that statins have immunosuppressive and immunomodulatory 119 applications[4, 5]. Fibrates (bezafibrate, ciprofibrate, fenofibrate and gemfibrozil) are prescribed 120 as first-line therapy in patients with severe hypertriglyceridaemia[6]. ARIs trigger a host

121 inflammatory immune response and can cause excessive secretion of pro-inflammatory cytokines

122 resulting in a cytokine storm, which can increase the risk of complications and mortality. Statins 123 and fibrates could potentially interfere with molecular pathways in influenza infection, reducing 124 secretion of pro-inflammatory cytokines, reducing inflammation, limiting the onset of a cytokine 125 storm and thus potentially reducing morbidity and mortality[7, 8]. These properties of statins and

126 fibrates could be advantageous in the clinical treatment and management of ARIs especially as

127 they have been used in clinical practice for years and have well established safety profiles. 
128 Therefore the aim of this study was to investigate the association between two lipid lowering 129 drugs, statins and fibrates, and all-cause 30-day mortality following a medically attended acute 130 respiratory illness (MAARI).

131

132

133

\section{Methods}

135

\section{Data source}

This study uses data obtained from the Clinical Practice Research Datalink (CPRD), a large population based observational and interventional research service providing anonymised UK primary care patient data. General practices that choose to participate in CPRD are required to record all instances of clinical diagnoses, morbidity events, abnormal test results and therapeutic information including prescription information, dosages and methods of administration. Furthermore, additional information is also recorded, such as weight, height, blood pressure measurements and lifestyle factors [9][9][9][9], making it the ideal data source for this study. Ethical approval for research involving CPRD data for this study was obtained from the CPRD Independent Scientific Advisory Committee (ISAC) (ISAC Protocol Number: Protocol 11_14R).

\section{Study design and population}

The CPRD data represent a primary care patient cohort and this study was conceptualised as a retrospective cohort study, in which nested case-control and survival analyses were conducted to investigate the research question (Figure 1). The source population for the present study consisted of all patients registered with general practices contributing to CPRD. 
158 Following a preliminary exploration of prescribing patterns for statins and fibrates, the study 159 period was defined as $1^{\text {st }}$ January 2008 to $31^{\text {st }}$ December 2013 . All participants aged 30 years and 160 older with a record of MAARI (Supplementary Appendix 1) within the study period were 161 included in the present study sample and within this group, patients classified as current statin 162 users based on prescription records were identified. It is assumed that all patients in the study 163 sample that were treated with statins, were clinically indicated for satin prescription (most likely 164 for primary or secondary cardiovascular disease prevention) and that statin prescription implies 165 the use of statins. Those without statin prescription were assumed to be non-users.

166 The most recent MAARI diagnosis date was used for each patient and even though MAARI 167 could recur during the study period, each patient was only counted once in the study. The start of 168 the 30-day follow up began from the MAARI index date (date of MAARI event as recorded by 169 the physician).

\section{Data variables}

172 The individual's exposure to either statins or fibrates in the 30 days prior to the MAARI episode 173 index date was coded as separate binary variables (yes/no). The outcome of interest was all174 cause mortality occurring in the 30 days following the MAARI index date. From a list of

175 covariates related to both the exposure and outcome of interest, we evaluated the following 
176 comorbidities as potential confounders: myocardial infarction, heart failure, peripheral vascular

177 disease, chronic lung disease and hypertension (all coded as dichotomous variables). In addition

178 we adjusted for total comorbidity burden using a weighted Charlsons's comorbidity index[10].

179 The CCI scores thus derived were further categorised into 4 levels $(0,1-2,3-5,>5)$ for inclusion

180 in the multivariable analysis as a categorical variable. We adjusted for the following drug

181 covariates: glitazones, metformin, beta blockers, ACE inhibitors and angiotensin receptor

182 blockers (ARBs). Finally, we included age, sex, body mass index (BMI) and smoking status as

183 socio-demographic and lifestyle variables, while the presence of an HbA1c measurement was

184 used as a proxy measure of healthcare seeking behaviour (Supplementary Appendix 1 includes

185 detailed variable definitions). For chronic conditions, we considered a diagnosis of that particular

186 condition at any point prior to the MAARI episode index date. For drug covariates, only current

187 exposures were considered. 'Current' was defined as the most recent prescription in the 30 days

188 prior to the MAARI index date.

189 These covariates were selected for adjustment based on a combination of what other researchers

190 had suggested/used, consultation with clinicians and clinical indications as per the British

191 National Formulary and NICE guidelines. [11, 12]

192 Given that statin prescription is a choice, apart from the medical indications for statin therapy, 193 certain behavioural factors may be related to statin use. Factors related to statin use such as 194 underlying cardiovascular comorbidity are easier to record, measure and adjust for. However, 195 behavioural factors and lifestyle preferences related to statin use are more difficult to measure 196 accurately. We adjust for the covariates that we have been able to measure (described above). 197 We discuss our findings in the context of those variables that we may not have been able to 198 measure and adjust for. 
199 For variables with $<5 \%$ missing data, a complete case analysis approach was adopted; for

200 variables with $>5 \%$ of missing data, a dummy variable was created to represent missing data.

201 Analysis

202 Descriptive analysis to summarise data characteristics, identify potential anomalies and quantify

203 missing data was conducted. To assess the average treatment effect across patients treated with 204 statins and fibrates, multivariable logistic regression and Cox regression models were 205 constructed to investigate the association between statins and fibrates and 30-day mortality 206 following MAARI. Proportional hazard assumptions were checked using log-log plots and the 207 Schoenfeld global test. Collinearity was assessed using the variance inflation factor. An a priori 208 decision was taken to include age, sex and current metformin and glitazone use (based on 209 previously reported immunomodulatory activity and likelihood of co-prescription for diabetes 210 mellitus[13]) in all multivariable models regardless of statistical significance. The models were 211 constructed as follows: Model A included a priori variables, all drug covariates, all comorbidity 212 variables, Charlson's comorbidity index (CCI) scores and socio-demographic and lifestyle 213 variables. Model B included a priori variables, and variables independently associated 214 (statistically significant at $\mathrm{P} \leq 0.05$ ) with both outcome and exposure. Model $\mathrm{C}$ included a priori 215 variables and variables that were both significantly $(\mathrm{P} \leq 0.05)$ associated with 30 -day mortality 216 and changed the crude measure of effect by $\geq 10 \%$. Results are presented as odds ratios (OR), 217 hazard ratios (HR) and 95\% confidence intervals (CI). Effect modification was assessed using 218 the likelihood ratio test and Model C was re-run stratified by significant interaction terms. 219 Additionally, we performed a sensitivity analysis where we adjusted for the number of GP visits 220 (included as a covariate) in each of the three models- A, B and C. 
221 All analyses were carried out in Stata 13 (StataCorp. 2009. Stata Statistical Software: Release 11.

222 College Station, TX: StataCorp LP).

223 Results

224 The final analysis sample after excluding patients aged 30 years or younger was 201,179 who 225 had a MAARI episode from 2008 to 2013. Of the study population 200,083 (95\%) survived at 226 the end of 30-day follow up, of which $40.8 \%$ were males and had a median age of 52 . Of the 227 surviving group, 27,095 were statin users and 611 were currently using fibrates. Crude analysis 228 showed a significant increased association between statin exposure and 30-day mortality [crude 229 OR: 1.55 (95\% CI: 1.34-1.81)] (table 1). All disease variables were significantly associated with 23030 -day mortality as were all socio-demographic and lifestyle variables.

231

232 All three multivariable logistic regression models yielded statistically significant point estimates 233 ranging from 0.63 to 0.67 as shown in table 2 . There was no effect modification of the 234 association between statins and 30-day mortality by either gender or BMI. However, a 235 significant interaction was found for CCI scores and therefore, in line with the analysis strategy, 236 Model C was re-run stratified by CCI score categories. The results of stratification showed point 237 estimates ranged from 0.48 to 0.63 but with overlapping 95\% confidence intervals (table 3 ).

238 The proportional hazards assumption was fulfilled as determined using log-log plots and the 239 Schoenfeld global test. Crude analysis found an increase in the hazard for 30-day mortality of $24058 \%$ in the statin users group [crude HR: 1.58 (95\% CI 1.36- 1.83)]. Multivariable Cox 241 proportional hazard regression analyses were conducted using the previously outlined 242 multivariable model building strategy and yielded significant point estimates ranging from 0.66 243 to 0.70 (table 4$)$. 
245 Sensitivity analysis

246 Statin users in our study sample were more likely $(\mathrm{p}<0.001)$ to consult GPs than non-users (Table 1$)$. We

247 performed a sensitivity analysis where we adjusted for number of GP consultations in our multivariable

248 models. After adjustment of number of GP visits, we obtained very similar estimates to our primary

249 results: Model A, OR (95\% CI): 0.67 (0.54 to 0.84); Model B: 0.63 (0.51 to 0.78); Model C: 0.65 (0.52 to

250 0.80). All three estimates were statistically significant $(\mathrm{p}<0.001)$.

251

252 Discussion

253 Summary of main findings

254 The findings of this study suggest that the use of statins is associated with decreased mortality in 255 the 30 days following a MAARI. Following adjustments for myocardial infarction, hypertension, 256 heart failure, diabetes, Charlson's comorbidity index and BMI (fully adjusted model C), current 257 statin use was protective and decreased the odds of MAARI-related mortality by $30 \%$. Cox 258 regression analysis showed a similar decrease in 30-day mortality hazard among statin users of $25935 \%$. We found a significant interaction for the effect of statins with comorbidity as measured 260 using the Charlson's comorbidity index and while stratification showed significant protective 261 effects of statins in the lower comorbidity categories, the $95 \%$ confidence intervals were 262 overlapping suggesting no clinically meaningful effect modifications. Fibrate use was found to 263 decrease MAARI-related mortality, but the results were non-significant.

264

265 Strengths and limitations

266 The validity of this study is enhanced by the quality, comprehensiveness and representativeness 267 of the data recorded within CPRD[9]. Additionally, the large sample exceeds a priori sample 268 size estimates (supplementary appendix 3), therefore increasing statistical power and reducing 
269 type II errors. Moreover, utilisation of contemporary data makes the findings potentially

270 applicable to current prescribing patterns. The retrospective cohort design of this study

271 eliminates temporal bias and there is no risk of recall bias as data are prospectively added to

272 CPRD and there is no risk of reverse causation as the outcome of death is final. It is however,

273 possible that miscoded entries due to user error may cause systematic errors and could result in

274 non-differential misclassification bias, pushing findings towards the null hypothesis.

275 All General Practitioner (GP) issued prescriptions are recorded within the database thus 276 minimising misclassification bias. While prescriptions issued in secondary care facilities are not 277 accounted for in CPRD, long-term medication for chronic conditions like statins, fibrates, 278 glitazones and metformin are mostly prescribed in primary care. Moreover, prescriptions are a 279 proxy measure of drug use and assume patient compliance. Lack of compliance would lead to 280 misclassification of exposure status and lead to an over-estimation of the association observed 281 and push findings towards the null hypothesis. The present study assumes patient compliance to 282 prescriptions based on indirect evidence that most patients do take prescribed drugs especially 283 long-term medication [14]. Moreover, this study assumes that those without a prescription for 284 statins are non-users, however it is possible that individuals who have a clinical indication for 285 receiving stations but refuse treatment have been omitted from the study sample and could 286 therefore overestimate the measure of effect. Furthermore, this study assumes that although there 287 may be differences in patients who were prescribed statins and those who were not, i.e. 288 confounding by indication leading to an overestimation of the observed effect, these differences 289 would be accounted for in the multivariable regression models.

290 Selection bias is minimised due to the method of data collection in CPRD, however a possible 291 limitation of CPRD data analysis is that MAARI patients presenting directly to secondary 
292 institutions (and presumably, more severe cases) may not be included, and therefore, these

293 findings may only be applicable to those with less severe MAARI that can be managed in 294 primary care.

295

296 Finally, it is not possible to prove a causal relationship between statin use and MAARI related 297 mortality based on an observational study; to strengthen the causal inference we adjusted for a 298 variety of drug, disease, socio-demographic and lifestyle covariates. However, an important 299 limitation with observational studies is that of residual confounding and omitted variable bias. It 300 is possible that, despite our attempts to adjust for relevant confounders, incorrect measurement of 301 a particular independent variable or omission of an unknown confounding variable could affect 302 our estimates. We attempted to overcome confounding by indication by estimating propensity 303 scores. However, our propensity scores could be limited by potentially omitted variables.

304 We earlier stated that there may be behavioural factors related to statin use that we may not have 305 been able to measure and subsequently adjust for in our analysis. There is evidence to suggest 306 that statin users are more likely to adopt a healthcare seeking lifestyle resulting in them being 307 healthier than non-users [15]. This 'healthy user effect' (i.e. statin users are healthier than non308 users) has been proposed as one likely explanation for previously observed statin-related benefits 309 in infection. However in our study sample, statin users were significantly more likely to have a 310 history of hypertension, myocardial infarction, heart failure, peripheral vascular disease, chronic 311 lung disease and higher number of comorbidities; statin users were also more likely to be current 312 smokers and obese (supplementary appendix 4). These factors explain why the crude effect 313 estimates showed a higher likelihood of MAARI-related mortality in statin users. In line with 314 previous evidence [15], statin users in our study sample were more likely to visit their 
315 physicians. However, when we adjusted for the number of GP visits, our estimates did not

316 change substantially. It is also important to note that the increased number of GP visits among

317 statin users may reflect genuine sicker patients with increased healthcare needs rather than being

318 indicative of a healthier lifestyle and it is unlikely that we have been able to fully account for the

319 healthy user effect. However, this study doesn't account for people who may have had elevated

320 cholesterol, but were contraindicated for statin therapy because they were likely to experience

321 side effects, not likely to take or adhere to prescriptions or were too frail. Patient frailty, although

322 difficult to measure in study, has not been accounted for, contributing to residual confounding

323 and should be taken into consideration when interpreting the findings from this study. Finally,

324 we have not performed instrumental variable analysis, a method described in the literature [16]

325 to account for non-random treatment assignment in observational studies investigating treatment

326 effects, in this current study; this is a limitation of this study.

327

328

329 Comparison with previous work

330 Statins and mortality

331 Existing research concurs with the protective association of statin use and MAARI-related

332 mortality results from this study $[17,18]$. Similarly, data from the Centre for Disease Control and

333 Prevention (CDC) influenza hospitalisation surveillance system[19], showed a similar yet more

334 conservative protective effect was reported, [adj. OR $0.59(95 \%$ CI $0.38-0.92)$ ] and could be due

335 to Vandermeer et al adjusting for age and comorbidity variables in-line with those included in

336 the present study as well as, influenza vaccination status and race; additional variables

337 unaccounted for in this study. Moreover, Vandermeer and colleagues considered patients 
338 requiring hospitalisation for influenza hence were more seriously ill and therefore the capacity to

339 benefit is likely to have been greater. Similarly, Myles et al reported a significant reduction in

340 pneumonia-related mortality among current statin users using data extracted from The Health

341 Improvement Network (THIN), a UK primary healthcare database similar to CPRD and could

342 not find evidence for the 'healthy user effect'. Finally a Canadian retrospective cohort study [20]

343 linking multiple administrative health-care databases over a 10 year period found borderline

344 protective effects of statin exposure in relation to 30-day mortality following influenza diagnosis

345 [crude OR: $0.92(95 \%$ CI $0.89-0.95)$ ] and a larger protective effect following pneumonia

346 diagnosis [crude: OR 0.84 (95\% CI 0.77-0.91)]; however both estimates shifted towards the null

347 following adjustment. This could be due to misclassification of exposure status as statin use was

348 not captured during hospitalisation of patients.

349 One randomised controlled trial performed in intensive care units in France, investigating the

350 effect of simvastatin treatment on mortality in patients with ventilator-associated pneumonia

351 reported no significant difference in 28-day mortality between the statin and the placebo groups

352 [Hazard Ratio (HR): 1.45 (95\% CI; 0.83 to 2.51)] [21]. Another trial performed in hospitalised

353 patients with community-acquired pneumonia showed no significant difference in time from

354 hospital admission to clinical stability between patients treated with simvastatin and placebo

355 [median: 3 days (IQR: 2 to 5) vs 3 days (IQR: 2 to 5); p-value: 0.47)] [22]. The 2012 JUPITER

356 trial studied the effect of rosuvastatin treatment on incident pneumonia in healthy patients

357 reported a modest benefit of statin treatment on the incidence of pneumonia [23].

358 While previous observational studies have reported a protective effect of statins, evidence from

359 randomised controlled trials show little of no effect of statins on pneumonia. One explanation for

360 this difference in estimates could be that the observational studies described above were carried 
361 out mostly using primary care datasets, whereas the trials have been carried out in intensive care

362 units and hospitalised patients where patients were demonstrably more ill. Polgreen et al., in their

363 cohort study investigating the effect of statins on pneumonia, have tried to minimise limitations

364 of an observational study design by performing instrumental variable analysis to account for

365 non-random assignment of statin treatment [16]. They reported that while statins were associated

366 with a reduction in pneumonia incidence in their initial analysis, this protective effect of statins

367 was not seen after accounting for non-random statin assignment indicating that the protective

368 effect seen initially was most likely due to healthy user effect [16].

369 The findings in this study suggest that statins may confer mortality-reduction benefits in patients

370 with MAARI. However, it should be noted that observational studies alone cannot prove a

371 conclusive causal relationship between statin exposure and decreased 30-day mortality following

372 MAARI and associations observed may be attributable to residual confounding; therefore, the

373 findings of this study should be considered in the context of other studies across different

374 populations and animal studies when considering the clinical implications. Nonetheless, the

375 observed mortality reduction among statin users are biologically plausible as their

376 immunomodulatory action has been demonstrated in animal and laboratory studies [5, 24-26]

377 and therefore, they could benefit MAARI patients by mediating their immune response.

378 However, prior to widespread use in this context, especially among patients in whom statins

379 would not otherwise be clinically indicated, randomised control trials are required to confirm

380 these potential benefits. It would also be valuable to investigate whether the mortality reduction

381 benefits vary for the different types of statins, the duration of statin exposure and, explicit

382 categorical definitions of statin dosage. 


\section{References}

385

386

387

1. Legand, A., S. Briand, and N. Shindo, Addressing the Public Health Burden of Respiratory Viruses. Future Virology, 2014. 8(10):953-968.

388

2. $\quad$ Kwak, B., et al., Statins as a newly recognized type of immunomodulator. Nat Med, 2000. 6(12): p. 1399-

389 1402.

390

3. Vaughan, C.J., M.B. Murphy, and B.M. Buckley, Statins do more than just lower cholesterol. The Lancet, 1996. 348(9034): p. 1079-1082.

392 4. Mach, F., Statins as immunomodulators. Transplant Immunology, 2002. 9(2-4): p. 197-200.

393

5. Raggatt, L. and N. Partridge, HMG-CoA Reductase Inhibitors as Immunomodulators. Drugs, 2002. 62(15):

394 p. $2185-2191$.

395

6. Miller, D. and J.D. Spence, Clinical Pharmacokinetics of Fibric Acid Derivatives (Fibrates). Clinical Pharmacokinetics, 1998. 34(2): p. 155-162.

7. Fedson, D.S., Pandemic Influenza: A Potential Role for Statins in Treatment and Prophylaxis. Clinical

8. Jain, M.K. and P.M. Ridker, Anti-Inflammatory Effects of Statins: Clinical Evidence and Basic Mechanisms. Nat Rev Drug Discov, 2005. 4(12): p. 977-987.

401

9. Herrett, E., et al., Validation and validity of diagnoses in the General Practice Research Database: a systematic review. Br J Clin Pharmacol, 2010. 69(1): p. 4-14.

403

10. Schneeweiss, S. and M. Maclure, Use of comorbidity scores for control of confounding in studies using

404 administrative databases. International Journal of Epidemiology, 2000.

405

406

407

11. British National Formulary. Statins. 2014; Available from:

https://www.evidence.nhs.uk/formulary/bnf/current/2-cardiovascular-system/212-lipid-regulatingdrugs/statins.

408

409

12. NICE clinical guideline 181. Lipid Modification-Cardiovascular risk assessment and the modification of blood lipids for the primary and secondary prevention of cardiovascular disease. 2014; Available from: https://www.nice.org.uk/guidance/cg181.

411

412

413

414

415

14. Jick, S.S., et al., Validity of the general practice research database. Pharmacotherapy, 2003. 23(5): p. 686-

15. Brookhart, M.A., et al., Adherence to Lipid-lowering Therapy and the Use of Preventive Health Services:

13. Fedson, D.S., Confronting the next influenza pandemic with anti-inflammatory and immunomodulatory agents: why they are needed and how they might work. Influenza and Other Respiratory Viruses, 2009. 3(4): p. 129-142.

418 16. Polgreen, L.A., et al., Increased statin prescribing does not lower pneumonia risk. Clinical Infectious 419 Diseases, 2015. 60(12): p. 1760-1766. 
420 17. Frost, F.J., et al., Influenza and COPD Mortality Protection as Pleiotropic, Dose-Dependent Effects of 421 Statins. CHEST Journal, 2007. 131(4): p. 1006-1012.

422

423

424

425

426

427

428

429

430

431

432

433

434

435

436

437

438

439

440

441

442

443

444

445

446

447

448

449

450

451

452

453

454

455

456

457

458

459

460

18. Myles, P.R., et al., The impact of statins, ace inhibitors and gastric acid suppressants on pneumonia mortality in a UK general practice population cohort. Pharmacoepidemiology and Drug Safety, 2014. 18(8): p. 697-703.

19. Vandermeer, M.L., et al., Association Between Use of Statins and Mortality Among Patients Hospitalized With Laboratory-Confirmed Influenza Virus Infections: A Multistate Study. The Journal of Infectious Diseases, 2011.

20. Kwong, J.C., P. Li, and D.A. Redelmeier, Influenza morbidity and mortality in elderly patients receiving statins: a cohort study. PloS one, 2009. 4(11): p. e8087.

21. Papazian, L., et al., Effect of statin therapy on mortality in patients with ventilator-associated pneumonia: A randomized clinical trial. JAMA, 2013. 310(16): p. 1692-1700.

22. Viasus, D., et al., The effect of simvastatin on inflammatory cytokines in community-acquired pneumonia: a randomised, double-blind, placebo-controlled trial. BMJ Open, 2015. 5(1).

23. Novack, V., et al., The effect of rosuvastatin on incident pneumonia: results from the JUPITER trial.

Canadian Medical Association Journal, 2012. 184(7): p. E367-E372.

24. Liao, J.K. and U. Laufs, PLEIOTROPIC EFFECTS OF STATINS. Annual Review of Pharmacology and Toxicology, 2005. 45(1): p. 89-118.

25. Farmer, J.A., Pleiotropic effects of statins. Current atherosclerosis reports, 2000. 2(3): p. $208-217$.

26. Davignon, J., Beneficial cardiovascular pleiotropic effects of statins. Circulation, 2004. 109(23 suppl 1): p. III-39-III-43.

(1)

3

4

(4)


461

462

463

464

465

466 


\section{Table 1 (on next page)}

Comparison of patient characteristics among non-statin users and current statin users 
Table 1: Comparison of patient characteristics among non-statin users and current statin users

\begin{tabular}{|c|c|c|c|c|}
\hline Patient Characteristic & $\begin{array}{c}\text { Non-statin } \\
\text { users }(n=174,084)\end{array}$ & $\begin{array}{c}\text { Current statin } \\
\text { users }(n=27,095)\end{array}$ & $\begin{array}{c}\text { Unadjusted } \mathrm{OR}^{1}(95 \% \\
\text { Confidence Interval) }\end{array}$ & P value ${ }^{2}$ \\
\hline Median Age (IQR) ${ }^{3}$ & $49(39-63)$ & $69(60-77)$ & $1.06(1.06$ to 1.07$)$ & $<0.001$ \\
\hline \multicolumn{5}{|l|}{ Sex } \\
\hline Males & $68,108(39.1 \%)$ & $13,962(51.5 \%)$ & 1 & \\
\hline Females & $105,976(60.9 \%)$ & $13,133(48.6 \%)$ & $0.60(0.59$ to 0.62$)$ & $<0.001$ \\
\hline Hypertension & $29,086(16.2 \%)$ & $13,377(49.4 \%)$ & $4.86(4.73$ to 4.99$)$ & $<0.001$ \\
\hline Myocardial Infarction & $3,134(1.9 \%)$ & $3,774(13.9 \%)$ & $8.34(7.94$ to 8.75$)$ & $<0.001$ \\
\hline Heart Failure & $1,410(0.8 \%)$ & $958(3.5 \%)$ & $4.49(4.13$ to 4.88$)$ & $<0.001$ \\
\hline Peripheral Vascular Disease & $1,076(0.6 \%)$ & $991(3.7 \%)$ & $6.10(5.59$ to 6.66$)$ & $<\mathbf{0 . 0 0 1}$ \\
\hline Chronic Lung Disease & $31,689(18.2 \%)$ & $6,041(22.3 \%)$ & $1.29(1.25$ to 1.33$)$ & $<0.001$ \\
\hline Diabetes & $10,162(5.8 \%)$ & $8,901(32.9 \%)$ & $7.89(7.64$ to 8.15$)$ & $<0.001$ \\
\hline \multicolumn{5}{|l|}{ Charlson's Comorbidity Score } \\
\hline 0 & $135,609(77.9 \%)$ & $10,315(38.1 \%)$ & 1 & \\
\hline $1-2$ & $29,257(16.8 \%)$ & $10,707(39.5 \%)$ & 4.81 (4.67 to 4.96$)$ & \\
\hline $3-5$ & $7,021(4.0 \%)$ & $4,476(16.5 \%)$ & $8.39(8.03$ to 8.75$)$ & \\
\hline$>5$ & $2,197(1.3 \%)$ & $1,597(5.9 \%)$ & $9.56(8.93$ to 10.22$)$ & $<0.001$ \\
\hline Fibrates & $363(0.2 \%)$ & $248(0.9 \%)$ & $4.42(3.76$ to 5.20$)$ & $<0.001$ \\
\hline Glitazones & $233(0.1 \%)$ & $690(2.6 \%)$ & $19.50(16.80$ to 22.63$)$ & $<0.001$ \\
\hline Metformin & $2,009(1.2 \%)$ & $4,608(17.0 \%)$ & $17.55(16.63$ to 18.53$)$ & $<0.001$ \\
\hline Beta Blockers & $3,452(2.0 \%)$ & $4,310(15.9 \%)$ & 9.35 (8.92 to 9.80$)$ & $<0.001$ \\
\hline ARB & $4,056(2.3 \%)$ & $4,089(15.1 \%)$ & $7.45(7.12$ to 7.80$)$ & $<0.001$ \\
\hline \multicolumn{5}{|l|}{ Smoking status } \\
\hline Never-smoker & $21,812(18.7 \%)$ & $2,627(14.7 \%)$ & 1 & \\
\hline Ex-smoker & $66,505(57.4 \%)$ & $8,475(47.4 \%)$ & $1.06(1.01$ to 1.12$)$ & \\
\hline Current-smoker & $27,654(23.9)$ & $6,765(37.9 \%)$ & 2.03 (1.94 to 2.13$)$ & $<0.001 *$ \\
\hline \multicolumn{5}{|l|}{$\mathrm{BMI}^{4}$} \\
\hline Underweight & $2,433(2.3 \%)$ & $251(1.5 \%)$ & 1 & \\
\hline Normal weight & $38,461(36.5 \%)$ & $3,817(22.2 \%)$ & $0.97(0.84$ to 1.10$)$ & \\
\hline Overweight & $37,522(35.6 \%)$ & $6,815(39.6 \%)$ & 1.77 (1.55 to 2.02$)$ & \\
\hline \multirow[t]{2}{*}{ Obese } & $26,983(25.6 \%)$ & $6,295(36.7 \%)$ & 2.27 (1.99 to 2.59$)$ & $<0.001^{*}$ \\
\hline & & & Mean difference $(95 \% \mathrm{CI})$ & p-value \\
\hline $\begin{array}{l}\text { Mean number of GP } \\
\text { consultations (SD) }\end{array}$ & $213.06(165.25)$ & $376.82(229.27)$ & $163.76(-166.01$ to 161.52$)$ & $<0.001$ \\
\hline
\end{tabular}




\section{Table 2 (on next page)}

Odds ratio's representing the association of statin exposure and 30-day mortality following MAARI 
Table 2. Odds ratio's representing the association of statin exposure and 30-day mortality following MAARI

\begin{tabular}{|c|c|c|c|}
\hline & $\mathbf{O R}^{1}$ & $95 \% \mathrm{CI}$ & P-Value \\
\hline Crude & 1.55 & 1.34 to 1.81 & $<0.001$ \\
\hline Model A* & 0.67 & 0.54 to 0.83 & $<0.001$ \\
\hline Model B & 0.63 & 0.51 to 0.78 & $<0.001$ \\
\hline Model C $C^{\ddagger}$ & 0.65 & 0.52 to 0.80 & $<0.001$ \\
\hline \multicolumn{4}{|c|}{$\begin{array}{l}{ }^{1} \text { Odds Ratio, }{ }^{2} \text { Confidence Interval } \\
\text { *Adjusted for a priori confounders, all comorbidity variables, all drug covariate variables, all potential } \\
\text { confounding variables } \\
\text { I Adjusted for a priori confounders, variables significantly associated with both outcome and exposure }(\leq 0.05) \\
\neq \text { Adjusted for a priori confounders, variables significantly associated with both outcome and exposure and } \\
\text { altering the crude OR by } \geq 10 \% \\
\text { Variables included in models A, B and C detailed in supplementary appendix } 2 \\
\text { Significant P- values shown in bold }\end{array}$} \\
\hline
\end{tabular}




\section{Table 3(on next page)}

The association between statins and 30-day mortality following MAARI: stratification by Charlson's Comorbidity Index categories 
Table 3. The association between statins and 30-day mortality following MAARI: stratification by Charlson's Comorbidity Index categories

\begin{tabular}{cccc}
\hline & Adjusted $\mathbf{O R}^{\mathbf{1}}$ & $\mathbf{9 5 \%} \mathbf{C I}^{\mathbf{2}}$ & $\mathbf{P}$ value \\
\hline Comorbidity index score $\mathbf{0}$ & 0.63 & 0.45 to 0.89 & $\mathbf{0 . 0 0 8}^{\ddagger}$ \\
Comorbidity index score 1-2 & 0.57 & 0.43 to 0.79 & $\mathbf{0 . 0 0 1}$ \\
Comorbidity index score 3-5 & 0.48 & 0.43 to 0.79 & $<\mathbf{0 . 0 0 1}$ \\
Comorbidity index score $>\mathbf{5}$ & 0.73 & 0.48 to 1.09 & 0.126 \\
\hline
\end{tabular}

${ }^{1}$ Odds Ratio, ${ }^{2}$ Confidence Interval

${ }^{\ddagger}$ LRT $p$ value

1

Significant $\mathrm{p}$ values shown in bold 


\section{Table 4 (on next page)}

Hazard ratios representing the association between statin exposure and 30- day mortality following MAARI 
Table 4. Hazard ratios representing the association between statin exposure and 30day mortality following MAARI

\begin{tabular}{|c|c|c|c|}
\hline & $H_{R^{1}}$ & $95 \%$ CI & P Value \\
\hline Crude & 1.58 & 1.36 to 1.83 & $<0.001$ \\
\hline Model A* & 0.70 & 0.57 to 0.83 & 0.001 \\
\hline Model B & 0.66 & 0.53 to 0.81 & 0.001 \\
\hline Model C C & 0.67 & 0.55 to 0.83 & $<0.001$ \\
\hline \multicolumn{4}{|c|}{$\begin{array}{l}{ }^{1} \text { Hazard Ratio, }{ }^{2} \text { Confidence Interval } \\
* \text { Adjusted for a priori confounders, all comorbidity variables, all drug covariate variables, all potential } \\
\text { confounding variables } \\
\text { i Adjusted for a priori confounders, variables significantly associated with both outcome and exposure } \\
(\leq 0.05) \\
\neq \text { Adjusted for a priori confounders, variables significantly associated with both outcome and exposure and } \\
\text { altering the crude HR by } \geq 10 \% \\
\text { Variables included in models A, B and C detailed in supplementary appendix } 2 \\
\text { Significant } p \text { values shown in bold }\end{array}$} \\
\hline
\end{tabular}

1 
1

Diagrammatic representation of retrospective cohort study design

Open Cohort

Study start 2008

30 day follow up

Exposed to

statins or fibrates in the30-days prior to MAARI

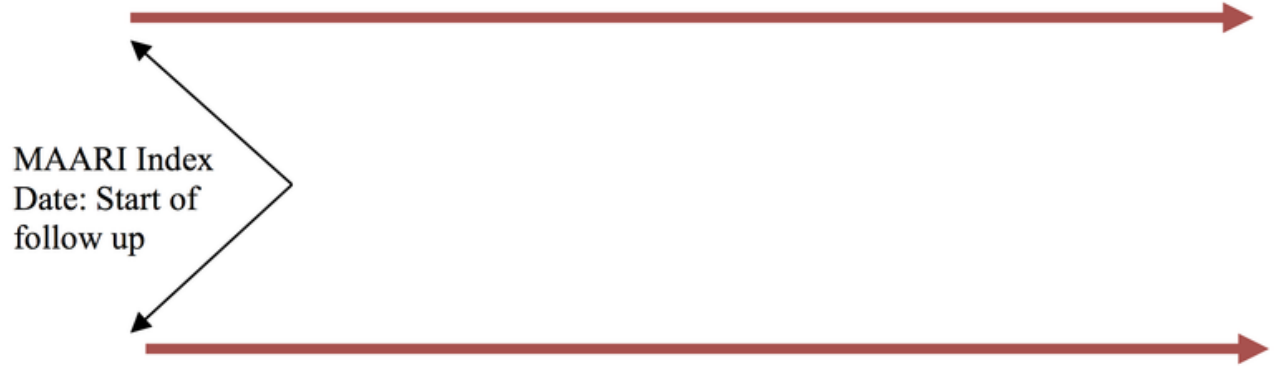

Unexposed to statins or fibrates in the 30-days

prior to MAARI

Exposure status ascertained at start of a patient's entry into the cohort; exposure ascertained with reference to index date;

All comorbidities ascertained before index date; records of comorbidity at any time in the patient's history before the index date were considered (on the assumption that chronic conditions will persist for the patient's lifetime)

All other drug exposures defined as 'current use' i.e. a prescription in the 30 days prior to the index date

Measurements such asHbA1c, BMI: the most recent record prior to the index date will be considered. 